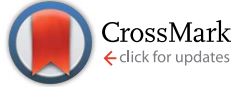

Cite this: RSC Adv., 2017, 7, 3992

Received 21st October 2016 Accepted 18th November 2016

DOI: $10.1039 /$ c6ra25645g

www.rsc.org/advances

\section{Tailoring the delivery of therapeutic ions from bioactive scaffolds while inhibiting their apatite nucleation: a coaxial electrospinning strategy for soft tissue regeneration $\dagger$}

\author{
Pin Zhou, ${ }^{a}$ Jian Wang, ${ }^{a}$ Anthony L. B. Maçon, ${ }^{\text {tb }}$ Akiko Obata, ${ }^{c}$ Julian R. Jones ${ }^{d}$ \\ and Toshihiro Kasuga*abc
}

The delivery of therapeutic ions, as a key element for the regeneration of soft tissue, represents a viable alternative to conventional drugs. Primarily designed for the regeneration of hard tissue, degradable bioactive inorganic matrices are a carrier of choice for the delivery of ionic chemical cues. However, they nucleate calcium-phosphate crystal on their surface, which could be undesired for most soft tissue regeneration. Here, a coaxial electrospinning process was engineered, generating core-shell fibres with inorganic particles enclosed within a bio-inert polymeric shell. Silicon doped vaterite (SiV) dispersed in poly(L-lactic acid) was selected as an inorganic composite core and poly(D,L-lactide-co-glycolide) (PLGA) as a shell. By careful selection of the electrospinning parameters, fibres of constant diameter $(\approx 10 \mu \mathrm{m})$ with controllable shell thickness (from 1.3 to $4.2 \mu \mathrm{m}$ ) were obtained. The release of calcium and silica followed the Weibull model, showing a purely diffusive release after hydration of the PLGA layer. The rate of release could be controlled with the shell thickness. The nucleation of calcium-phosphate crystals was inhibited. In addition, with the presence of a PLGA shell layer, the mechanical properties of the fibermats were greatly improved with, for instance, an increase of the Young's modulus up to 536\% as compared to original composite. These non-woven porous materials are an affordable investigation platform to study the effect of local ionic release onto the surrounding cell metabolism.

\section{Introduction}

Recently new biomaterials have been developed that can deliver therapeutic ions for the regeneration of soft tissues. ${ }^{1-3}$ Enriching the local environment of human cells in ions such as calcium or silicate ions can have significant effects on their metabolism, activating genetic pathways, which can subsequently accelerate the recovery of damaged tissues. ${ }^{4-7}$ For instance, calcium plays a central role in wound healing as it is involved in various cellular processes triggered by cutaneous injuries. ${ }^{8,9}$ In addition, internalised calcium is known to regulate inflammatory cell infiltration and favour the proliferation of fibroblasts. ${ }^{10,11}$ Few examples are available in the literature,

\footnotetext{
${ }^{a}$ Department of Frontier Materials, Nagoya Institute of Technology, Gokiso-cho, Showa-ku, Nagoya 4668555, Japan. E-mail: kasuga.toshihiro@nitech.ac.jp ${ }^{b}$ Frontier Research Institute for Materials Science, Nagoya Institute of Technology, Gokiso-cho, Showa-ku, Nagoya 4668555, Japan. E-mail: alb.macon@nitech.ac.jp 'Division of Advanced Ceramics, Nagoya Institute of Technology, Gokiso-cho, Showaku, Nagoya 4668555, Japan

${ }^{d}$ Department of Materials, Imperial College London, South Kensington Campus, London SW7 2AZ, UK

$\dagger$ Electronic supplementary information (ESI) available. See DOI: $10.1039 / \mathrm{c} 6 \mathrm{ra} 25645 \mathrm{~g}$
}

demonstrating that a biomaterial-based approach is a viable strategy for the local delivery of calcium in wounded skin..$^{12-15}$ Kawai et al. developed calcium-based nanoparticles from a fetal bovine serum that can readily disintegrate in acidic $\mathrm{pH}$, delivering ionised calcium. Intravenous injection in female Balb/c mice revealed a significant increase in the resorption of the wound as compared to the control. ${ }^{15}$

In the wider context of soft tissue regeneration, bioactive ceramics and glasses can deliver ions that have therapeutic properties. ${ }^{3,16}$ Ionic chemical cues can be present within the structure of the inorganic construct either as network modifier/ former for bioactive glasses or as a part of the crystal lattice for bioceramics and can be released upon hydrolytic degradation when immersed in body fluid. ${ }^{17}$ However, most of the engineered bioactive glasses and ceramics have been designed towards the regeneration of hard tissue. Thus, the release of these active ions often comes with a great variation in surface chemistry, favouring the surface nucleation of bone-like crystal, an essential step in the osteoconduction cascade. ${ }^{18}$

While this is considered to be a desirable point in the regeneration of hard tissues, it may be a source of complications in regeneration of soft-tissues as it can lead to calcification, in particular for cardiovascular tissues. ${ }^{19}$ In this report, we 
present a proof-of-concept for the fabrication of open 3D composite templates, allowing the release of inorganic chemical cues from bioactive glasses or ceramics while inhibiting the formation a hydroxyapatite layer on the surface of the template. A coaxial electrospinning setup was used to create a polymeric core-shell non-woven fabric within which the inorganic particles were loaded in the core of the fibres protected by a polymeric shell. ${ }^{20}$ One important criterion of this engineered system is that the shell layer must uptake water from the surrounding body fluid in order to trigger the hydrolytic degradation of the inorganic particles and subsequent diffusion of the therapeutic ions in the media.

In order to validate this proof of concept, we selected siliconcontaining vaterite (SiV) as an inorganic phase. SiVs are nonthermodynamically stable calcium carbonate spherical particles of approximately $1 \mu \mathrm{m}$ which can release calcium and organo-silicate ions upon immersion in aqueous media. ${ }^{21-24}$ When directly exposed to aqueous media, full conversion into calcite is observed within $1 \mathrm{~h}$ with a dissolution rate tailorable as a function of the synthesis parameters. Poly(lactic acid) was selected as an organic phase for the core of the fibres since this composite has already been successfully electrospun. ${ }^{25-28}$ Poly(D,L-lactide-co-glycolide) (PLGA) was selected has an outer layer for its ability to uptake water and therefore allowing the hydrolytic degradation of the enclosed SiV particles. ${ }^{\mathbf{2 0}, 29,30}$ The effect of the relative diameter of the inner core to the size of the PLGA shell on the mechanical properties, dissolution behaviour, and apatite nucleation was investigated.

\section{Experimental section}

\subsection{Materials}

Poly(L-lactic acid) (PLLA, Mitsui Chemicals, Co., Ltd. Japan; $M_{\mathrm{w}}$ $=140 \mathrm{kDa})$, poly (D,L-lactide-co-glycolide) (PLGA, Purasorb ${ }^{\circledR}$ PLGA, Purac Biomaterials) and siloxane-containing vaterite (SiV, Yabashi Industries, Co., Ltd. Japan, $2.6 \mathrm{wt} \%$ of silicon, 1.4 $\mu \mathrm{m}$ in diameter) were used as received. All other chemicals were purchased from Wako Pure Chemical Industries, Japan.

\subsection{Preparation of core-shell composite fibres by coaxial electrospinning}

SiVPC (SiV/PLLA composite) was prepared by a melt-kneading method. ${ }^{25}$ PLLA was poured into a preheated kneader and stirred for $5 \mathrm{~min}$ at $200{ }^{\circ} \mathrm{C}$, and then mixed with $\mathrm{SiV}$ and stirred for another $10 \mathrm{~min}$. The mass ratio of SiV to PLLA was set to $60 \mathrm{wt} \%$.

Coaxial electrospinning equipment (Kato Tech, NEU, Japan) was used to prepare the core-shell composite fibres. SiVPC (10 $\mathrm{wt} \%$ ) and PLGA (15 wt \%) were separately dissolved in chloroform and stirred at room temperature for $12 \mathrm{~h}$. Each solution was loaded into separate syringes and set up on a pump (FP-W-100, Melquest, Toyama, Japan). The syringes were connected to the coaxial needles (inner diameter $\emptyset_{\text {in }}=0.50 \mathrm{~mm}$, outer diameter $\emptyset_{\text {out }}=1.10 \mathrm{~mm}$ ) to form a concentric nozzle, with PLGA and SiVPC representing the shell and the core of the electrospun fibres, respectively. The drum collector, with a tangential velocity of $2 \mathrm{~m} \mathrm{~min}^{-1}$ wrapped in aluminium foil, was placed at 150-200

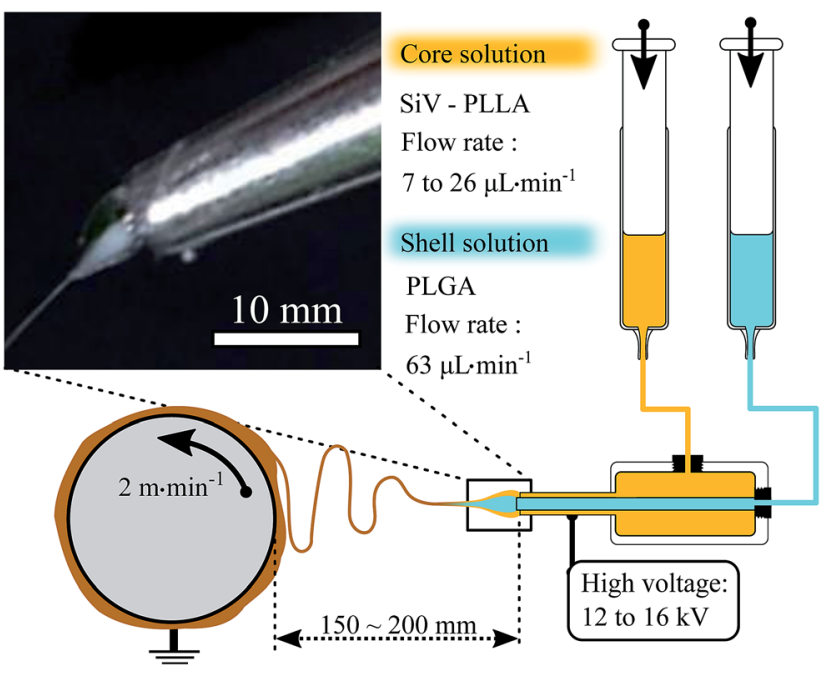

Fig. 1 Schematic representing the coaxial electrospinning setup used here to produce core-shell fibres with a photograph of the concentric spinneret (inset).

$\mathrm{mm}$ from the nozzle. The electrospinning was carried out applying a of $+12 \mathrm{kV}$ potential between the nozzle and the collector for $4 \mathrm{~h}$, at room temperature with a relative humidity of $\approx 50 \%$. A schematic of the setup is shown in Fig. 1 .

The extrusion rate of the SiVPC shell was set to $63 \mu \mathrm{L} \mathrm{min}{ }^{-1}$. The extrusion rate of the core layer (SiVPC) was set relatively to the shell at $2 \times(\mathrm{CS}-1)$ and $10 \times(\mathrm{CS}-2)$ slower. Conventional fibres were also prepared using the above method and a single needle from SiVPC and pure PLGA dissolved in chloroform at $13 \mathrm{wt} \%$ and $15 \mathrm{wt} \%$, respectively.

\subsection{Characterisation of core-shell composite fibres}

Morphologies of the surfaces and the fracture surfaces of the fibres were observed with a scanning electron microscope (SEM, JSM-6301F, JEOL, Japan) after coating with amorphous osmium. The diameters of core-shell fibres were measured from 40 randomly chosen fibres using Image J software. The cross sections were observed by soaking the fibres in liquid nitrogen for $2 \mathrm{~min}$ and breaking with tweezers. Tensile test was conducted on an Autograph (AGS-G, Shimadzu, Japan). The experiment followed the Japanese Industrial Standard JIS L1015. All samples were cut using a metal punch $(50 \mathrm{~mm} \times 10$ $\mathrm{mm})$. The grip-to-grip distance was $40 \mathrm{~mm}$ and the samples were elongated at a constant tensile rate of $1 \mathrm{~mm} \mathrm{~min}^{-1}$ until failure. Each composition was run in triplicate.

\subsection{Ion release behaviour}

The ion release behaviour of the fibres was evaluated as previously described $^{23}$ in Tris buffer solution (TBS). Briefly, $1000 \mathrm{~mL}$ of TBS was prepared by dissolving $6.118 \mathrm{~g}$ of tris(hydroxymethyl) aminomethane in distilled water at $36.5^{\circ} \mathrm{C}$, then adjusting $\mathrm{pH}$ to 7.4 with $1 \mathrm{M}$ hydrochloric acid. $20 \mathrm{mg}$ of sample was placed in polypropylene containers, subsequently filled with $10 \mathrm{~mL}$, tightly sealed, and kept in a incubation oven at $36.5{ }^{\circ} \mathrm{C}$ in static state. At each time point ( 3 h, 6 h, 12 h, 3 d, 7 d and $10 \mathrm{~d}$ ), samples were 
taken out from the solution, rinsed with DW and dried overnight. The concentrations of silica and calcium ions in the residual solutions were measured by inductively coupled plasma atomic emission spectroscopy (ICP-AES; ICPS-7000, Shimadzu, Japan). The ICP-AES was calibrated prior to use using calcium and silicon standard solutions at 2, 10, $40 \mu \mathrm{g} \mathrm{mL} \mathrm{m}^{-1}$. Each time point was run in triplicate for statistical relevance.

\subsection{Apatite-forming ability}

Simulated body fluid (SBF, $\mathrm{pH}=7.4)^{31}$ consisting of $142.0 \mathrm{mM}$ $\mathrm{Na}^{+}, 5.0 \mathrm{mM} \mathrm{K}, 1.5 \mathrm{mM} \mathrm{Mg}^{2+}, 2.5 \mathrm{mM} \mathrm{Ca}^{2+}, 148.3 \mathrm{mM} \mathrm{Cl}^{-}$, $4.2 \mathrm{mM} \mathrm{HCO}^{3-}, 1.0 \mathrm{mM} \mathrm{HPO}_{4}{ }^{2-}, 0.5 \mathrm{mM} \mathrm{SO}_{4}{ }^{2-}$ was prepared by dissolving reagent grade $\mathrm{NaCl}, \mathrm{NaHCO}_{3}, \mathrm{KCl}, \mathrm{K}_{2} \mathrm{HPO}_{4} \cdot 3 \mathrm{H}_{2} \mathrm{O}$, $\mathrm{MgCl}_{2} \cdot 6 \mathrm{H}_{2} \mathrm{O}, \mathrm{HCl}, \mathrm{CaCl}_{2}$, and $\mathrm{Na}_{2} \mathrm{SO}_{4}$ in distilled water at $36.5{ }^{\circ} \mathrm{C}$, and then using tris(hydroxymethyl) aminomethane and $1 \mathrm{M}$ hydrochloric acid to adjust $\mathrm{pH}$ to $7.4 .20 \mathrm{mg}$ of samples was immersed in $10 \mathrm{~mL}$ of SBF and kept at $37^{\circ} \mathrm{C}$ in static state for 1 or 3 days. Samples were then washed with distilled water and dried in air.

For characterisation of the crystalline phases, an X-ray diffraction (XRD, X'pert X-ray Diffractometer, Philips) analysis was conducted $\left(\mathrm{CuK}_{\alpha}, 50 \mathrm{kV}, 40 \mathrm{~mA}\right)$. The scan rate was $0.01^{\circ} \mathrm{s}^{-1}$ and a $2 \theta$ range was from $20^{\circ}$ to $60^{\circ}$. Before tests, the dried sample were cut to $10 \mathrm{~mm} \times 20 \mathrm{~mm}$.

\section{Results and discussion}

\subsection{Preparation of the core-shell composite fibres varying the shell wall thickness}

Fig. 1 shows the experimental setup used to obtain non-woven core-shell fibres composed of $\mathrm{SiV}$ particles embedded in poly(L-lactic acid) for the inner fibre and poly(D,L-lactide ${ }_{75}-\mathrm{CO}$ glycolide $_{25}$ ) for the shell. Coaxial-electrospinning consists of applying a high-voltage to concentric needles from which two solutions of different compositions were fed. The variables affecting the coaxial-electrospinning were conceptually similar to these of single jet. ${ }^{32}$ However, key parameters had to be carefully selected in order to achieve an uniform coating of the sheath polymer onto the core fibres, which can be found in Table $1:^{33}$ (i) the working range of applied voltage used was found to be between 12 to $16 \mathrm{kV}$, guaranteeing the formation of a single Taylor cone comprising both the core and the shell as shown the inset of Fig. 1. Moghe et al. described that a subcritical voltage could lead to a single jet of sheath polymer, whereas a supercritical voltage could lead to multiple jets, spinning separately the core and the shell. ${ }^{33}$ However, this phenomenon was not observed here. (ii) The stabilisation of the Taylor cone was obtained due to the low interfacial tension between the core and shell solutions as chloroform was used as solvent for both solutions, which also favoured the formation of uniform core-shell fibres. ${ }^{34}$ (iii) Intermixing of the core and shell solutions was avoided by assuring that the difference in viscosity between two liquids was sufficient, and that the flow rate of the core solution was at least twice lower than the sheath solution. ${ }^{35}$ (iv) Finally, the flow rate of the core solution must be high enough in order to facilitate the formation of an inner jet and uniform fibres without beading. ${ }^{36}$ Here, a fixed flow rate of $63 \mu \mathrm{L} \min ^{-1}$ was selected for the shell solution, while varying flow rate of the from 6.8 (CS-2) to 26.4 (CS-1) $\mu \mathrm{L} \mathrm{min}^{-1}$, expecting an increase of the inner diameter and overall fibre diameters as an increase of the core solution flow rates. ${ }^{33,36,37}$

Fig. 2 shows SEM pictures of the fibres and their crosssections after the electrospinning process, from which the characteristic sizes of the cores and shells were extracted and summarised in Table 1. Uniform fibres were produced with no apparent disruption of the core relative to the shell. Crosssectioning of the fibres revealed that the SiV particles were well confined within the core of the fibres, suggesting that no intermixing of the solution occurred during the electrospinning process. Interestingly, the overall diameter of the fibres, of an approximate value of $10 \mu \mathrm{m}$, did not vary by decreasing the flow rate of the core solution, as suggested elsewhere in the literature. ${ }^{36-38}$ Instead, the core diameter decreased from $7.4 \mu \mathrm{m}$ at 26.4

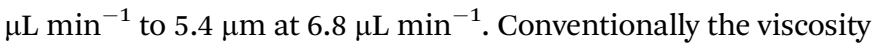
of the sheath solution is set higher than the core to facilitate the formation of an uniform Taylor cone, counterbalancing the interfacial tension. ${ }^{34,36,39}$ However, due to the composite nature of the core solution, this criterion could not be validated here, which nonetheless did not have detrimental impact on electrospinnability of the material. Thus, we hypothesised that when the differential viscosity between the sheath and core solution is inverted, the rapid drying of the sheath solution relative to the core induced a sudden increase of the interfacial tension between the two liquids, and as a result stress hardened the core fibres during the whipping process. The direct consequence was that

Table 1 Summary of the electrospinning parameters used to fabricate the core-shell composite fibres and their characteristic sizes ${ }^{a}$

\begin{tabular}{|c|c|c|c|c|c|c|c|}
\hline Entry & Ratio $^{\mathrm{a}}$ LA/GA & $\begin{array}{l}\text { Viscosity } \\
\text { (Pa s) }\end{array}$ & $\begin{array}{l}\text { Polymer content }{ }^{c} \\
(w t \%)\end{array}$ & $\begin{array}{l}\text { Feed rate } \\
\text { ratio }\end{array}$ & $\begin{array}{l}\text { Voltage }^{\mathrm{e}} \\
(\mathrm{kV})\end{array}$ & $\begin{array}{l}\text { Fibre } \emptyset^{\mathrm{f}} \\
(\mu \mathrm{m})\end{array}$ & $\begin{array}{l}\text { Wall thickness }{ }^{\mathrm{f}} \\
(\mu \mathrm{m})\end{array}$ \\
\hline SiVPC & $100 / 0$ & 4600 & 10 & - & 12 & $10 \pm 1$ & - \\
\hline CS-2 & $75 / 25$ & 2.1 & 15 & 9.2 & 12 & $10 \pm 2$ & $2.3 \pm 0.1$ \\
\hline CS-3 & $85 / 15$ & 2.5 & 15 & 2.4 & 15 & $12 \pm 2$ & $1.6 \pm 0.1$ \\
\hline CS-4 & $50 / 50$ & 2.2 & 13 & 2.4 & 16 & $11 \pm 3$ & $1.3 \pm 0.6$ \\
\hline
\end{tabular}

${ }^{a}$ a Molar ratio of lactic and glycolic acid in poly(D,L-lactide-co-glycolide); ${ }^{\mathrm{b}}$ obtained from the polymer/polymer-composites solubilised in $\mathrm{CHCl}_{3}$ as shown in ${ }^{\mathrm{c}}$; ${ }^{\mathrm{d}}$ sheath to core flow rate ratio with a shell set at $63 \mu \mathrm{L} \mathrm{min}{ }^{-1}$; ${ }^{\mathrm{e}}$ voltage applied between the coaxial needle and the collector; ${ }^{\mathrm{f}}$ measured from the SEM micrograph $n=40$. 

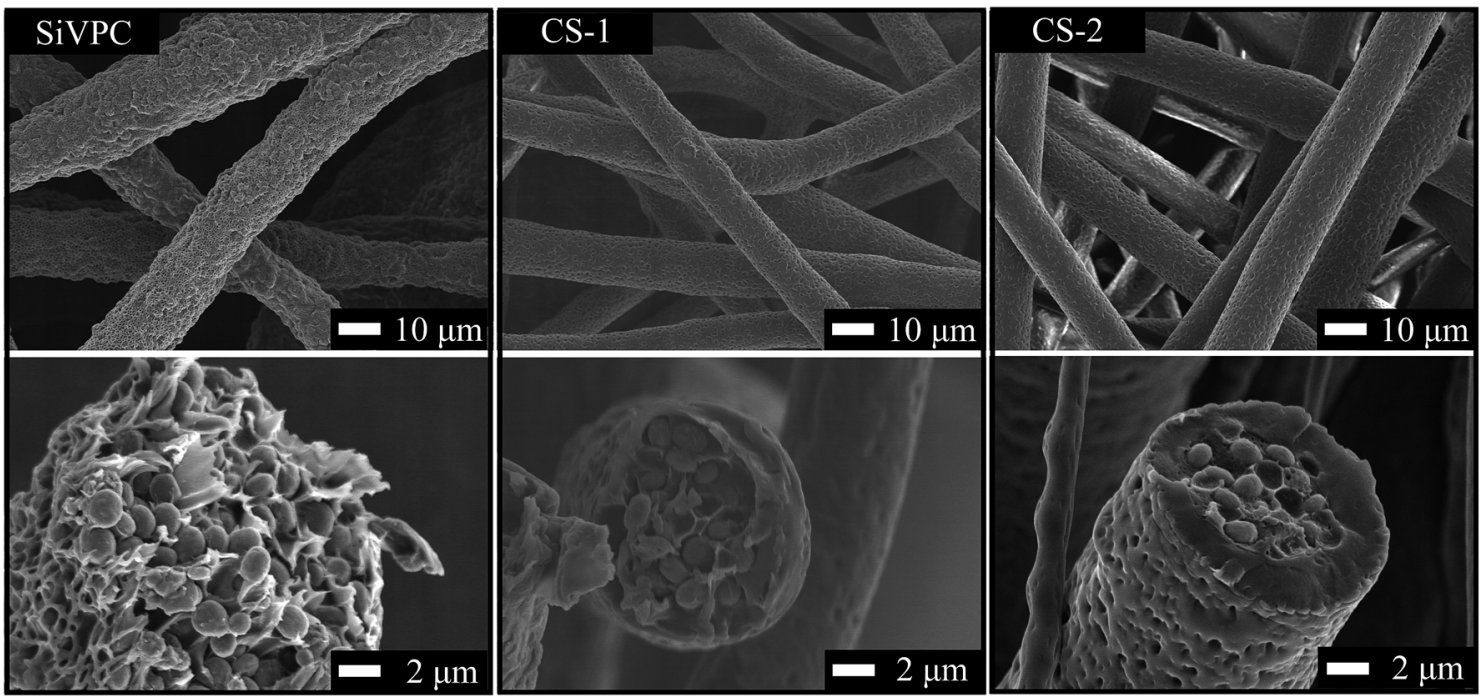

Fig. 2 SEM micrographs showing the porous structure of the core-shell fibres after electrospinning and their corresponding cross-section.

the core diameter could be controlled by its flow rate. In order to validate our observation, the experiment was replicated with a core solution flow rate 20 times lower than the sheath solution. Fibres with an overall diameter of $11 \pm 2 \mu \mathrm{m}$ and an inner diameter of $4.2 \pm 1 \mu \mathrm{m}$ were obtained (Fig. S1 $\dagger$ ), confirming the observation made above.

The morphology of the fibres were also characterised by SEM as shown in Fig. 2. Fibres exhibited rough surfaces, with potential porosity, regardless of the experimental condition used. Several reports on single jet electrospinning demonstrated that the surface morphology of fibres made from polyester is dictated by complex interactions between the polymer, solvent and the local environment in which process is conducted..$^{\mathbf{4 0 , 4 1}}$ Here, experiments were conducted at room temperature with a relative humidity of $50 \%$ and the morphology of the fibres were in good agreement with the these obtained by Putti et al. using poly( $(\varepsilon-$ caprolactone) in $\mathrm{CHCl}_{3}$, under the same conditions. ${ }^{\mathbf{4 0}}$

\subsection{Effect of the PLGA wall thickness on ion release}

In order to evaluate the ion release from the fibermats, $20 \mathrm{mg}$ of SiVPC, CS-1 and CS-2 were immersed in $50 \mathrm{mM}$ Tris buffered solution with $\mathrm{pH}$ adjusted to 7.4. The silicon and calcium release profiles are shown in Fig. 3a and were obtained by analysing the collected solution by ICP-AES. Calcium and soluble silica from SiVPC burst in solution with initial release rates of $R_{\text {SiVPC,Ca }}=27$ $\mu \mathrm{g} \mathrm{mL} \mathrm{m}^{-1} \mathrm{~h}^{-1}$ and $R_{\text {SiVPC,Si }}=8 \mu \mathrm{g} \mathrm{mL}^{-1} \mathrm{~h}^{-1}$ reaching $314.5 \pm 0.6$ $\mu \mathrm{g} \mathrm{mL}{ }^{-1}$ and $32.0 \pm 0.8 \mu \mathrm{g} \mathrm{mL}^{-1}$ after $1 \mathrm{~d}$ of immersion and 345.4 $\pm 5.1 \mu \mathrm{g} \mathrm{mL} \mathrm{m}^{-1}$ and $32.3 \pm 0.8 \mu \mathrm{g} \mathrm{mL}^{-1}$ at $3 \mathrm{~d}$ for calcium and silicon, respectively, and staying constant thereafter. Both ions were released in media due to hydrolytic instability of the silicon containing vaterite particles embedded within the PLLA matrix, with profile releases in agreement with our previous report. $22,26,28$ The initial release rate of the calcium was only 3.4 times higher than silicon, when silicon only represents $2.8 \%$ of the weight of the SiV particles. This means that the silicon release was relatively higher than calcium, which can be explained by the structural role that hydrolysed 3-aminopropyltriethoxysilane (APTES), the silicon source in SiV, plays in SiV particles. ${ }^{23}$ APTES stabilises the premature crystalline phases during the synthesis of SiV particles by enclosing them in a peripheral layer. Thus, upon immersion of the particles in aqueous media, oligomeric and monomeric aminopropylsilanetriols were first released in solution, in a burst fashion, subsequently followed by the release of calcium. ${ }^{23}$

With the presence of a poly(D,L-lactide ${ }_{75}-$ co-glycolide $\left.{ }_{15}\right)$ shell layer onto the SiVPC fibres, both initial rate of release and total amount release at 10 days were reduced with a more pronounced effect as the wall thickness increased. For instance, the calcium initial rate of release decreased to $R_{\mathrm{CS}-1, \mathrm{Ca}}=16 \mu \mathrm{g}$ $\mathrm{mL}^{-1} \mathrm{~h}^{-1}$ and to $R_{\mathrm{CS}-2, \mathrm{Ca}}=2 \mu \mathrm{g} \mathrm{mL} \mathrm{m}^{-1} \mathrm{~h}^{-1}$ with an wall thickness of 1.3 and $2.3 \mu \mathrm{m}$, respectively. The hydrolytic degradation of the embedded SiV particles, through the PLGA layer, was possible due to the water uptake of the shell layer. ${ }^{30,42}$

However, despite the apparent reduction in concentration in calcium and silica with the increase of the wall thickness, it is difficult to draw firm conclusion as an increase in wall thickness came with a decrease in SiV particle content at a fixed mass of fibres. Thus, to alleviate the uncertainty, the total content in silica and calcium per gram of fibres was evaluated after alkaline digestion, allowing the normalisation of the release profiles presented in Fig. 3a, as shown in Fig. 3b. This revealed that with an outer layer of $1.3 \mu \mathrm{m}$ (CS-1), the general release rate of silica and calcium was reduced compared to SiVPC, however, with no statistical decrease of the initial rate. For instance, calcium was initially released at $7.0 \pm 1 \% \mathrm{~h}^{-1}$ for SiVPC and $6.0 \pm 1 \% \mathrm{~h}^{-1}$ for CS-1. With an outer thickness of $2.3 \mu \mathrm{m}$, the initial release rate was $2.5 \pm 0.5$, approximately half of SiVPC. In addition, it appeared that the modal release for CS- 2 varied from CS-1 with a sustain release of calcium after $3 \mathrm{~d}$ immersion at a rate of release of $3 \% \mathrm{~d}^{-1}$. In order to verify whether this change in release behaviour was due to a change in mechanism of release or just a consequence of the increase in shell thickness, the profiles in Fig. 3b were fitted with the Weibull model, 
a)

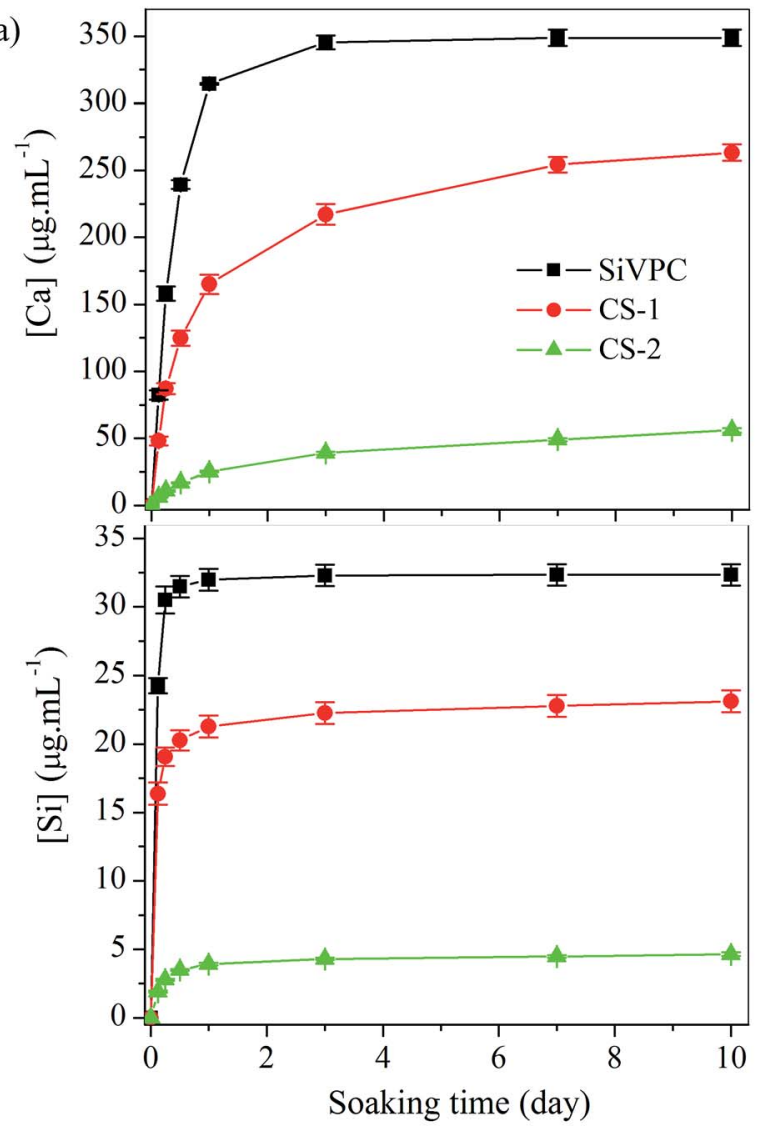

b)

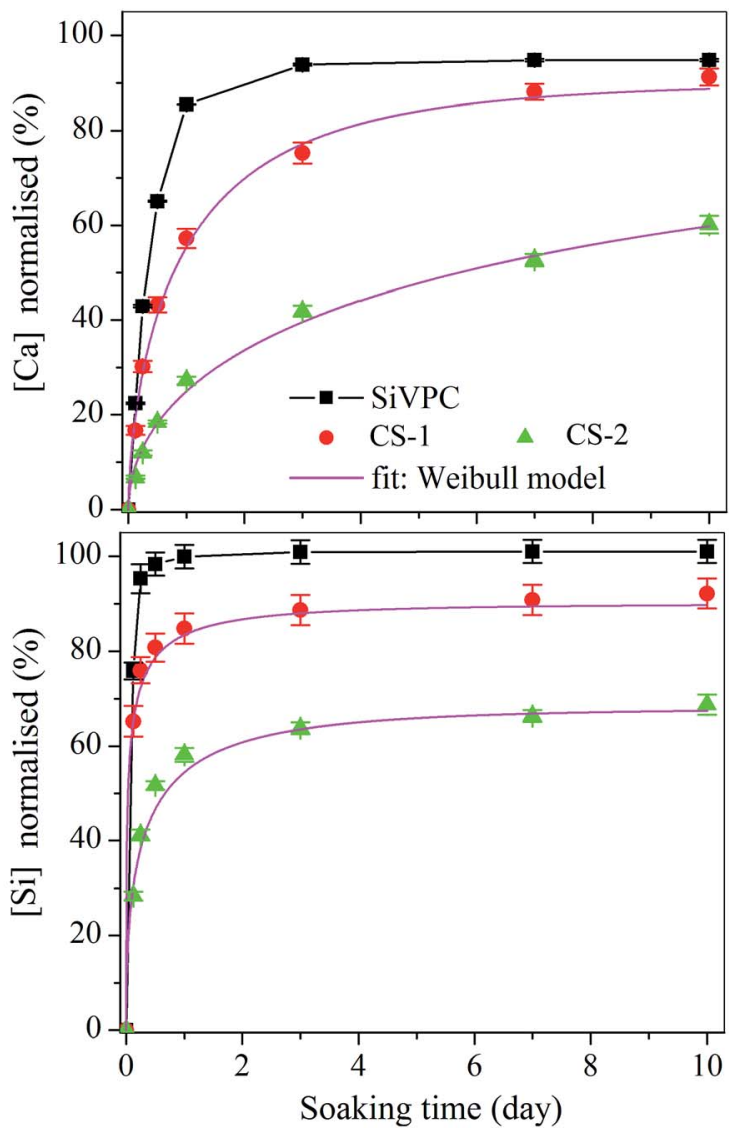

Fig. 3 Calcium and silicon release profiles upon immersion in Tris buffered solution over $10 \mathrm{~d}$ with (a) the concentration given in $\mu \mathrm{g} \mathrm{mL}^{-1}$ and (b) the normalised concentration. The normalised profiles of the core-shell fibres were fitted using eqn (1).

characterising a purely diffusive, or Fickian, ionic transport, using the following equation: ${ }^{43,44}$

$$
\frac{M_{t}}{M_{\infty}}=1-\exp \left(-a \times t^{b}\right)
$$

where $M_{\infty}$ is the total amount of released ions at infinity, $M_{t}$ the amount of ions released at $t, a$ and $b$ constants. All release profiles from the core-shell fibres could be fitted with the Weibull model with a $R^{2}$ above 0.99 , suggesting that the ionic release from the SiV particles through the PLGA layer was purely diffusive. In addition, when using PLGA as a template, the value taken by $b$ could inform on the mechanism of diffusional release and the degree of disordering of the medium (i.e. the PLGA shell). ${ }^{45,46}$ Since the same polymer was used as a shell for CS- 1 and CS-2, $b$ is only describing the change in release mechanism. Thus, with CS-2 $(b=0.354 \pm 0.01)$ the diffusion was characteristic of a percolation cluster whereas with CS-1 $(b$ $=0.954 \pm 0.05$ ) the diffusion followed a first order with regards to the Fick's law of diffusion, highlighting changes in release mechanism with the wall-thickness. In addition, this model suggests that increasing or decreasing the ordering of the template could also lead to variations in the ionic release profiles. Fortunately, the degree of ordering in PLGA and its ability to uptake water can be tuned as a function its chemical composition, by varying the relative content of lactic to glycolic residue in the copolymer. An increase in lactic content would lead in a more hydrophobic shell and vice versa. To verify the validity of this hypothesis, the core-shell fibres similar to CS-1 in geometry were produced using PLGA with a lactic to glycolic molar ratio of $85: 15$ (CS-3) and $50: 50$ (CS-4) (SEM

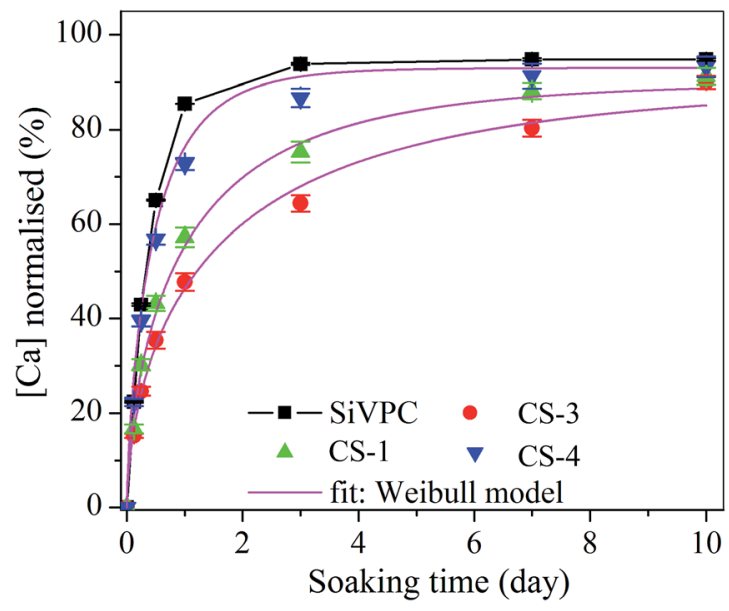

Fig. 4 Normalised calcium release profiles in Tris buffer solution from core-shell fibres of the same wall-thickness, varying the ratio of lactic to glycolic acid in the PLGA shell layer. The normalised profiles of the core-shell fibres were fitted using eqn (1). 
pictures available in ESI, Fig. S2 $\dagger$ ) and immersed in Tris buffered solution as shown in Fig. 4 (silicon release in Fig. S3†). CS-1 and SiVPC were plotted as a control. As expected the release behaviour of PLGA ( $85: 15)$ was slower than PLGA (75:25), which was itself higher than PLGA $(50: 50)$. The data were fitted with the Weibull model and showing an increase in the value $b$ with PLGA (50:50) to 1.515 and a decrease with PLGA (85:15) to 0.724 , indicating variations in the mechanism of release. ${ }^{\mathbf{4 5 , 4 6}}$ This highlights the complexity of ionic diffusion through PLGA membranes or shell but also shows the high degree of tailorability of the system. It is important to note that these release profiles could also be greatly influenced with the hydrolytic stability of the inorganic construct embedded with the core of the fibres and are only valid for SiV particles.

\subsection{Apatite-forming ability}

The precipitation of hydroxyapatite onto the surface of glasses or ceramics is known to be a surface nucleation driven process, where topography, surface chemistry and local environment play detrimental roles in the mechanism. ${ }^{18}$ By enclosing the SiVPC fibres with a bio-inert polymer, we hypothesised that crystal nucleation could be suppressed. ${ }^{47}$ To confirm this statement, SiVPC, CS-1 and CS-2 were immersed in simulated body fluid (SBF) over $3 \mathrm{~d}$ and the variation in surface chemistry was monitored by XRD and SEM. This measurement is not to be related to the potential in vivo performance of these non-woven fibres but to understand the change in chemistry upon immersion in media that have the same ionic strength that blood plasma. ${ }^{48}$
Fig. 5 shows the SEM micrographs of the fibermats before and after 1 and $3 \mathrm{~d}$ of immersion in SBF. After $1 \mathrm{~d}$, cauliflowerlike crystals of $\approx 1 \mu \mathrm{m}$ were partially covering the SiVPC fibres, which slightly grew in size and number after $3 \mathrm{~d}$ of immersion. The morphology of the crystals was typical of early stage nucleation of calcium-phosphate on bioactive materials. ${ }^{18}$ It is likely that the rapid increase in calcium concentration along with the rough surface of the fibres could have favoured the nucleation of calcium-phosphate crystal as SBF is supersaturated towards hydroxyapatite. ${ }^{49}$ However, it is unclear whether the polymer or the exposed SiV particles acted as nucleation centre. The presence of a shell layer significantly reduced or suppressed the crystal nucleation on the surface of the fibres. Upon immersion of CS-1, submicron particles could be observed on the surface of the fibres. However, it is difficult to appreciate whether these particles were formed by nucleation, or were $\mathrm{SiV}$ particles exposed by early erosion of the PLGA shell layer. With a wall thickness of $2.3 \mu \mathrm{m}$, such as with CS-2, no precipitate could be observed, with smooth fibres at 1 and 3 $\mathrm{d}$ of immersion, result of the shell hydration. ${ }^{30,42}$ Fig. 6 shows the XRD patterns before and after immersion in SBF for SiVPC, CS-1 and CS-2. SiV particles are characterised by diffraction peaks at $21^{\circ}$ and $25^{\circ}$ and $33^{\circ} 2 \theta$ (ICSD 18127), originating from the (004), (110) and (114) planes of their hexagonal unit cell, respectively. ${ }^{23}$ Before immersion, the intensity of diffracted peaks, corresponding to $\mathrm{SiV}$, decreased with an increase of the wall thickness. Clear diffraction at peak at $2 \theta \approx 32^{\circ}$ could be observed after $3 \mathrm{~d}$ of immersion for SiVPC, which is

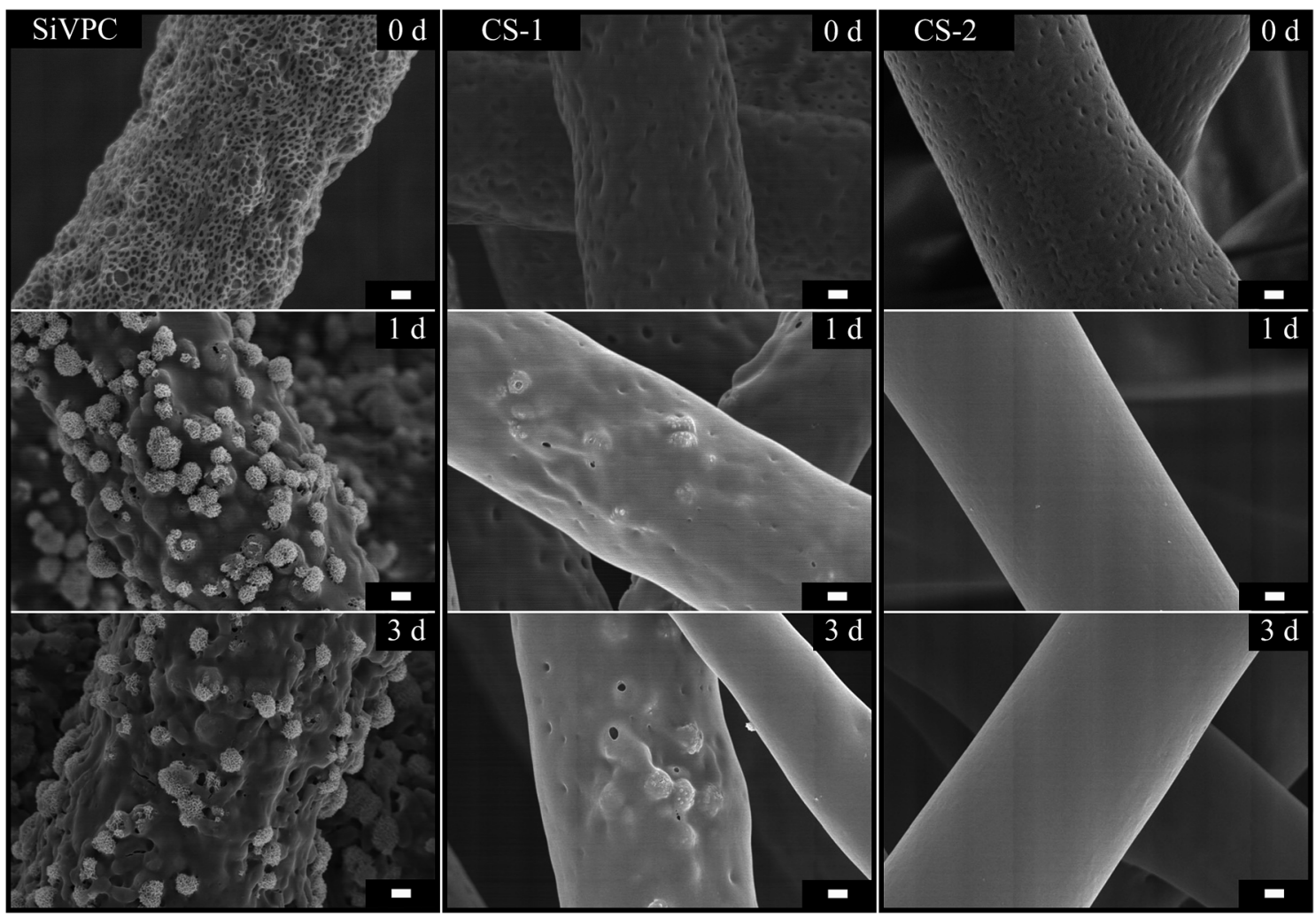

Fig. 5 SEM micrographs of SiVPC, CS-1 and CS-2 before and after immersed in SBF for 1 day and 3 days, the scale bar is $1 \mu$ m. 

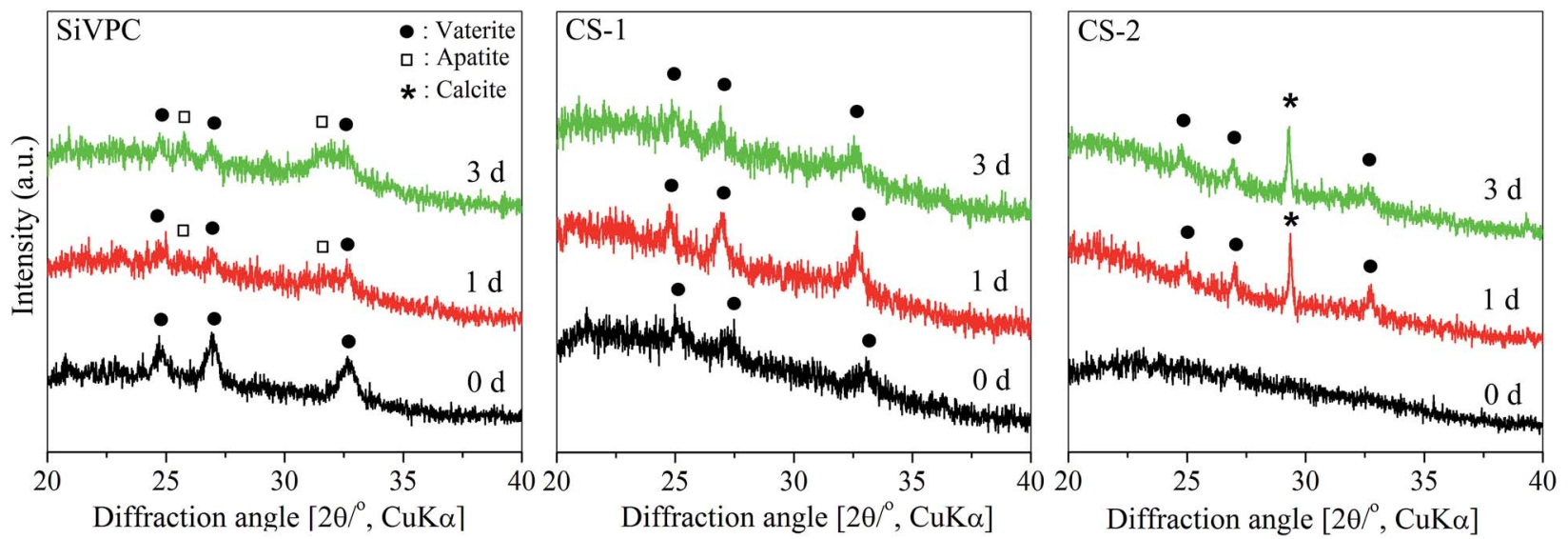

Fig. 6 XRD patterns of the core-shell fibres before and after $1 \mathrm{~d}$ and $3 \mathrm{~d}$ of immersion in simulated body fluid.

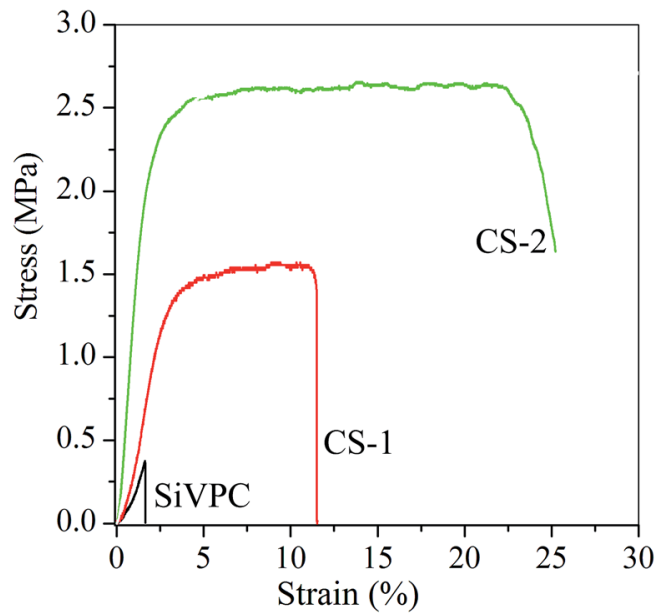

Fig. 7 Typical stress-strain curves obtained from tensile deformation of the fibremats.

Table 2 Summary of values extracted from the stress-strain curve in Fig. 7 describing the mechanical properties of the core-shell fibres. Values are given from a population of $n=3$

\begin{tabular}{llcc}
\hline Entry & $\begin{array}{l}\text { Tensile } \\
\text { strength }(\mathrm{MPa})\end{array}$ & $\begin{array}{l}\text { Elongation } \\
\text { at break }(\%)\end{array}$ & $\begin{array}{l}\text { Young's } \\
\text { modulus (MPa) }\end{array}$ \\
\hline SiVPC & $0.52 \pm 0.33$ & $1.77 \pm 0.79$ & $22 \pm 1$ \\
CS-1 & $1.78 \pm 0.39$ & $10.60 \pm 0.80$ & $49 \pm 1$ \\
CS-2 & $2.76 \pm 0.33$ & $25.52 \pm 7.89$ & $118 \pm 11$
\end{tabular}

characteristic of crystallised calcium-phosphate (ICSD 01-0841998) and corroborated the observations made with SEM. However, the definition of the peak was not sufficient to conclude with certainty that the crystal formed was hydroxyapatite. With CS-1, vaterite was the only crystalline phase detected, regardless of the immersion period whereas calcite was detected after $1 \mathrm{~d}$ of immersion for CS-2 (ICSD 52151). It is likely that with CS-2 the slow ionic diffusion along with the efficient hydration of the shell led to the conversion of SiV into calcite within the core of the fibres as no crystal could be seen on their surface.

\subsection{Mechanical properties of the core-shell composite fibres}

It is important that when synthesising synthetic materials for medical application, the resulting template has enough strength to be physically handled and manipulated by the surgeons without breaking. ${ }^{2}$ SiVPC is known to be brittle due to the high loading of inorganic particles (60 wt\%). ${ }^{26}$ Thus, additionally to the above proof of concept, we investigated whether the addition of a PLGA shell layer could have a beneficial effect onto the mechanical properties of the electrospun composites.

Tensile test was performed SiVPC, CS- 1, CS- 2 and the resulting stress-strain curves are shown in Fig. 7 and the extracted tensile strength, elongation at break and Young's modulus in Table 2. As expected, SiVPC fibermat was brittle with an elongation at break of $1.77 \pm 0.79 \%$. Upon the addition of a PLGA shell layer the mode of deformation went from brittle to ductile, with a proportional increase of the mechanical properties with the increase of the wall thickness. For instance, the Young's modulus of the fibermats, extracted for the elastic region, went from $22 \mathrm{MPa}$ for SiVPC to 118 for CS-2 along with $530 \%$ increase in the tensile strength and $1441 \%$ in elongation at break.

\section{Conclusion}

We successfully demonstrated that the apatite forming ability of bioactive inorganic construct could be suppressed while retaining the release of inorganic chemical cues in the surrounding media. This engineered strategy could opened new perspectives to the large library of inorganic construct that have been originally designed for the repair of hard tissue towards the regeneration of soft tissue, where calcification could lead to non-negligible complications. We also believe that these nonwoven porous materials could also be used as investigation platform to study the effect of local ionic release onto the surrounding cell metabolism. 


\section{Acknowledgements}

The present work was conducted in the framework of Academic Unit Cooperation Program between Nagoya Institute of Technology and Imperial College London, and was supported in part by JSPS Program for Advancing Strategic International Networks to Accelerate the Circulation of Talented Researchers/\#R2704.

\section{References}

1 V. Miguez-Pacheco, L. L. Hench and A. R. Boccaccini, Acta Biomater., 2015, 13, 1-15.

2 J. R. Jones, Acta Biomater., 2013, 9, 4457-4486.

3 M. N. Rahaman, D. E. Day, B. S. Bal, Q. Fu, S. B. Jung, L. F. Bonewald and A. P. Tomsia, Acta Biomater., 2011, 7, 2355-2373.

4 A. Hoppe, N. S. Gőldal and A. R. Boccaccini, Biomaterials, 2011, 32, 2757-2774.

5 A. L. B. Maçon, M. Jacquemin, S. J. Page, S. Li, S. Bertazzo, M. M. Stevens, J. V. Hanna and J. R. Jones, J. Sol-Gel Sci. Technol., 2016, DOI: 10.1007/s10971-016-4097-x.

6 A. Obata, Y. Takahashi, T. Miyajima, K. Ueda, T. Narushima and T. Kasuga, ACS Appl. Mater. Interfaces, 2012, 4, 56845690.

7 G. Poologasundarampillai, D. Wang, S. Li, J. Nakamura, R. Bradley, P. Lee, M. Stevens, D. McPhail, T. Kasuga and J. Jones, Acta Biomater., 2014, 10, 3733-3746.

8 S. K. Yoo, C. M. Freisinger, D. C. LeBert and A. Huttenlocher, J. Cell Biol., 2012, 199, 225-234.

9 J. V. Cordeiro and A. Jacinto, Nat. Rev. Mol. Cell Biol., 2013, 14, 249-262.

10 A. Jadali and S. Ghazizadeh, J. Biol. Chem., 2010, 285, 2338723397.

11 D. D. Bikle, D. Ng, C.-L. Tu, Y. Oda and Z. Xie, Mol. Cell. Endocrinol., 2001, 177, 161-171.

12 Y. Suzuki, Y. Nishimura, M. Tanihara, K. Suzuki, T. Nakamura, Y. Shimizu, Y. Yamawaki and Y. Kakimaru, J. Biomed. Mater. Res., Part A, 1998, 39, 317-322.

$13 \mathrm{~J}$. W. Doyle, T. P. Roth, R. M. Smith, Y. Q. Li and R. M. Dunn, J. Biomed. Mater. Res., 1996, 32, 561-568.

$14 \mathrm{~J}$. S. Boateng, K. H. Matthews, H. N. Stevens and G. M. Eccleston, J. Pharm. Sci., 2008, 97, 2892-2923.

15 K. Kawai, B. J. Larson, H. Ishise, A. L. Carre, S. Nishimoto, M. Longaker and H. P. Lorenz, PLoS One, 2011, 6, e27106.

16 A. El-Ghannam, Expert Rev. Med. Devices, 2005, 2, 87-101.

17 T. Kasuga, Acta Biomater., 2005, 1, 55-64.

18 A. L. B. Maçon, T. B. Kim, E. M. Valliant, K. Goetschius, R. K. Brow, D. E. Day, A. Hoppe, A. R. Boccaccini, I. Y. Kim, C. Ohtsuki, T. Kokubo, A. Osaka, M. Vallet-Regí, D. Arcos, L. Fraile, A. J. Salinas, A. V. Teixeira, Y. Vueva, R. M. Almeida, M. Miola, C. Vitale-Brovarone, E. Verné, W. Holand and J. R. Jones, J. Mater. Sci.: Mater. Med., 2015, 26, 115.

19 W. Karwowski, B. Naumnik, M. Szczepański and M. Myśliwiec, Med. Sci. Monit., 2012, 18, 1-11.

20 J. Wang, P. Zhou, A. Obata, J. R. Jones and T. Kasuga, Materials, 2015, 8, 7979-7987.
21 A. Obata, S. Tokuda and T. Kasuga, Acta Biomater., 2009, 5, 57-62.

22 J. Nakamura and T. Kasuga, J. Ceram. Soc. Jpn., 2013, 121, 792-796.

23 J. Nakamura, G. Poologasundarampillai, J. R. Jones and T. Kasuga, J. Mater. Chem. B, 2013, 1, 4446-4454.

24 S. Yamada, Y. Ota, J. Nakamura, Y. Sakka and T. Kasuga, J. Ceram. Soc. Jpn., 2014, 122, 1010-1015.

25 T. Wakita, J. Nakamura, Y. Ota, A. Obata, T. Kasuga and S. Ban, Dent. Mater. J., 2011, 30, 232-238.

26 K. Fujikura, S. Lin, J. Nakamura, A. Obata and T. Kasuga, J. Biomed. Mater. Res., Part B, 2013, 101, 1350-1358.

27 A. Obata, T. Hotta, T. Wakita, Y. Ota and T. Kasuga, Acta Biomater., 2010, 6, 1248-1257.

28 T. Wakita, A. Obata, G. Poologasundarampillai, J. R. Jones and T. Kasuga, Compos. Sci. Technol., 2010, 70, 1889-1893.

29 J. K. Perron, H. E. Naguib, J. Daka, A. Chawla and R. Wilkins, J. Biomed. Mater. Res., Part B, 2009, 91, 876-886.

30 H. K. Makadia and S. J. Siegel, Polymers, 2011, 3, 1377-1397.

31 T. Kokubo and H. Takadama, Biomaterials, 2006, 27, 29072915.

32 J. M. Deitzel, J. Kleinmeyer, D. Harris and N. C. B. Tan, Polymer, 2001, 42, 261-272.

33 A. K. Moghe and B. S. Gupta, Polym. Rev., 2008, 48, 353-377.

34 J. H. Yu, S. V. Fridrikh and G. C. Rutledge, Adv. Mater., 2004, 16, 1562-1566.

35 A. Townsend-Nicholson and S. N. Jayasinghe, Biomacromolecules, 2006, 7, 3364-3369.

36 M. Wang, J. H. Yu, D. L. Kaplan and G. C. Rutledge, Macromolecules, 2006, 39, 1102-1107.

37 H. Na, P. Chen, S.-C. Wong, S. Hague and Q. Li, Polymer, 2012, 53, 2736-2743.

38 Y. Z. Zhang, X. Wang, Y. Feng, J. Li, C. T. Lim and S. Ramakrishna, Biomacromolecules, 2006, 7, 1049-1057.

39 F. Elahi, W. Lu, G. Guoping and F. Khan, J. Bioeng. Biomed. Sci., 2013, 3, 1000121.

40 M. Putti, M. Simonet, R. Solberg and G. W. Peters, Polymer, 2015, 63, 189-195.

41 L. Huang, N.-N. Bui, S. S. Manickam and J. R. McCutcheon, J. Polym. Sci., Part B: Polym. Phys., 2011, 49, 1734-1744.

42 D. J. Hines and D. L. Kaplan, Crit. Rev. Ther. Drug Carrier Syst., 2013, 30, 257-276.

43 W. Weibull, J. Appl. Mech., 1951, 13, 293-297.

44 K. Kosmidis, P. Argyrakis and P. Macheras, J. Chem. Phys., 2003, 119, 6373.

45 V. Papadopoulou, K. Kosmidis, M. Vlachou and P. Macheras, Int. J. Pharm., 2006, 309, 44-50.

46 S. Giovagnoli, P. Blasi, M. Ricci, A. Schoubben, L. Perioli and C. Rossi, J. Pharm. Sci., 2008, 97, 303-317.

47 F. G. Torres, S. N. Nazhat, S. H. S. M. Fadzullah, V. Maquet and A. R. Boccaccini, Compos. Sci. Technol., 2007, 67, 11391147.

48 M. Bohner and J. Lemaitre, Biomaterials, 2009, 30, 21752179.

49 A. Oyane, H.-M. Kim, T. Furuya, T. Kokubo, T. Miyazaki and T. Nakamura, J. Biomed. Mater. Res., Part A, 2003, 65, 188195. 